\title{
Original
}

\section{Effect of Chewing Gum Containing Calcified Seaweed on Remineralization and Acid Resistance of Enamel Subsurface Lesions}

\author{
Yasuo Miake ${ }^{1)}$, Nobuhiko Hiruma ${ }^{1)}$, Shinya Asada²) and Akira katakura ${ }^{3)}$ \\ ${ }^{1)}$ Department of Ultrastructural science, Oral Science Center, Tokyo Dental College, Chiba, Japan \\ ${ }^{2}$ Oral Science Section, Basic Research Department, Central Laboratory, Saitama, Japan \\ 3) Department of Oral medicine, Oral and Maxillofacial Surgery, Tokyo Dental College,Chiba, Japan \\ (Accepted for publication, July 15, 2012)
}

\begin{abstract}
Calcified seaweed contains abundant quantities of calcium, magnesium, phosphate, sodium and fluorine ions. The objective of this study was to investigate the effect of chewing gum containing calcified seaweed on the remineralization and aciduric properties of enamel. For intraoral experiments, enamel blocks prepared from third molars were attached to an appliance. Each block was covered with acid-resistant nail varnish, except for a window measuring 6 x $4 \mathrm{~mm}$. A caries-like lesion was artificially prepared in the window. Participants attached the appliance and chewed gum for $20 \mathrm{~min}, 4$ times a day. The intraoral experiment period was 2 weeks. Half of each window was re-covered with nail varnish. In the aciduric experiment, these blocks were immersed in demineralizing solution. After demineralization, the blocks were embedded in polyester resin and prepared in ground sections. Contact microradiograms of these ground sections were obtained. A high degree of remineralization occurred at the surface and at a deep layer in the remineralized enamel area. In the aciduric experiment area, further demineralization was observed beneath the original demineralized layer, and overall depth of demineralization increased. A thin, highly mineralized layer was observed between the two demineralized layers. The degree of remineralization and aciduric rate with calcified seaweed treatment was higher than that with non-calcified seaweed treatment $(\mathrm{p}<0.05)$. These results suggest that chewing calcified seaweed-containing gum significantly enhances the remineralization and acid resistance of enamel by exposure to fluorine ions.
\end{abstract}

Key words: Caries prevention, Calcified seaweed, Enamel, Remineralization, Xylitol

\section{Introduction}

The enamel surface is stabilized through repeated demineralization and remineralization by saliva ${ }^{1}$. While eating food containing cariogenic sweeteners such as sucrose increases the acidity of saliva, saliva provides a buffering action against this acidity, immediately returning the oral environment to neutrality ${ }^{2,3}$. Saliva also shows bactericidal activity against cariogenic bacteria $^{4,5}$. Supersaturation by calcium (Ca) and phosphate ions (P) is essential in suppressing the onset of demineralization and inducing remineralization. In addition, a small quantity of fluorine ions (F) is also required ${ }^{6,7)}$. Thus, saliva plays an important role in the prevention of caries.

Chewing gum is known to have a beneficial effect on oral health and may be a useful adjunct to oral hygiene. Moreover, chewing gum acts as a potent stimulant of salivary flow, thus increasing

Correspondence to:Yasuo Miake DDS. PhD,Department of Ultrastructural Science, Tokyo Dental College, 1-2-2 Masago, Mihama-ku, Chiba 2618502, Japan. Tel.: +81-43-270-3755,Fax.: +81-43-270-3756, E-mail address: miake@tdc.ac.jp
$\mathrm{Ca}$ and $\mathrm{P}$ levels, limiting demineralization and enhancing remineralization ${ }^{8}$. An earlier study showed that an up to 10 -fold increase in salivary flow rate compared with the unstimulated rate occurred within the first minute of gum chewing ${ }^{9)}$. Chewing gum is an effective delivery vehicle for an agent as it involves contact between the agent and the teeth for protracted lengths of time and only minimal effort on behalf of the consumer. An earlier study evaluated potentially anticarious agents in chewing gum ${ }^{10)}$.

The gum used for this experiment included xylitol. Xylitol is a widely used non-cariogenic food additive. Xylitol has also been claimed to be a non-acidogenic sweetener that increases salivary secretion and the amount of calcium in plaque, but not acid production ${ }^{11)}$. In addition, it is thought to enhance the remineralization of initial carious lesions ${ }^{12)}$. Xylitol is believed to be associated with calcium in aqueous solution ${ }^{13}$ and inhibit the dissolution of calcium and phosphate ions from enamel ${ }^{14}$.

Fluoride and xylitol are used to enhance the effect of saliva ${ }^{15-}$ ${ }^{19)}$, and F, in particular, is highly effective in the prevention and recovery of initial carious lesions ${ }^{16)}$. However, not being 
J.Hard Tissue Biology Vol. 21(3):315-320, 2012

recognized as a food additive, $\mathrm{F}$ cannot be directly added to gum in Japan. In our previous study ${ }^{20,21)}$, we demonstrated that the abundant quantities of Ca, P and F contained in calcified seaweed enhanced the aciduric properties and remineralization of enamel.

The objective of this study was to investigate the effect of chewing gum containing calcified seaweed on the remineralization and aciduric properties of enamel in human tooth.

\section{Materials and Methods}

\section{Participants}

Seven healthy volunteer participants were selected from among the postgraduate students at Tokyo Dental College. Written informed consent was obtained from all participants prior to enrolment. The study protocol was approved by the Ethics Committee of Tokyo Dental College (Ethical Clearance Number 189). An intraoral examination was performed prior to experiments to confirm the absence of current carious activity, periodontal disease or other pathology.

\section{Preparation of enamel blocks}

Fourteen human third molars extracted due to pericoronitis from patients aged 20-40 years were used. All samples were preserved in $10 \%$ formalin solution. The study protocol was approved by the Ethics Committee of Tokyo Dental College. Each crown was cut to obtain three enamel blocks. The enamel surface of each enamel block was polished to a flat surface with wet abrasive paper of \#2000 to expose fresh enamel. For the intraoral experiments, a removable acrylic appliance was fabricated for each volunteer's lower jaw, covering the lingual surface from the first premolar to the second molar. The base of the appliance was held in place by 2 stainless steel clasps. Each enamel block was covered with acid-resistant nail varnish, except for an area measuring $6 \times 4 \mathrm{~mm}$ (experimental window). Two enamel blocks were attached to each appliance (orally used blocks, OB), and autoclave sterilization performed.

Next, caries-like lesions were artificially prepared in the window of each block by the method of White ${ }^{22}$ with modifications. Briefly, the appliance was immersed in $50 \mathrm{ml}$ demineralizing solution ( $0.1 \mathrm{M}$ lactic acid, $0.3 \mathrm{mM}$ hydroxyapatite, $1 \%$ carboxymethyl cellulose sodium salt, $\mathrm{pH} 4.7$ ) at $37^{\circ} \mathrm{C}$ for 3 days. After demineralization, the enamel blocks were washed with distilled water and dried. Each block was then re-covered with nail varnish (demineralization area, DA), but leaving two thirds of the window uncovered. For the aciduric experiments, in addition to blocks used in the intraoral experiments, some enamel blocks were demineralized but not used intraorally (non-orally used blocks, NOB). The appliance and enamel blocks were stored in a plastic tube in a humidified environment until used.

\section{Preparation of chewing gum containing calcified seaweed}

Calcified seaweed (Aquamineral $\mathrm{T}^{\circledR}$ : Biocon Japan Ltd.) was obtained from calcareous deposits of dead seaweed accumulated at the bottom of the sea. The calcified seaweed contained 34.0\% Ca, 3.14\% magnesium (Mg), 0.56\% P, 0.24\% sodium (Na), 0.11\% $\mathrm{F}$ and other components. The calcified seaweed was dissolved in acid due to its poor solubility. The solution was neutralized and freeze-dried to obtain a powder.

Two kinds of chewing gum including 36\% xylitol and 2\% citric acid were obtained from Lotte Co. Ltd: one containing 1.6\% calcified seaweed (CS group) and the other not (non-CS group).

\section{Experimental protocol}

The protocols of the two intraoral experiments were identical except for the composition of the chewing gum. The gums were provided to the participants as coded products and stored at room temperature. All chewing gum treatments were double-blinded and randomized. In each experiment, type of chewing gum was assigned at random to each participant.

Each intraoral experimental period lasted 2 weeks with a 1week rest between experiments. All participants used non-fluoride toothpaste for the duration of each experiment. In the intraoral experiment, after attaching the appliance, the participant chewed gum for $20 \mathrm{~min}, 4$ times a day (after breakfast, lunch and supper, and before retiring). After removing the gum, the appliance was preserved in the oral cavity for a further $20 \mathrm{~min}$. When the appliances were removed, they were stored in a plastic tube in a humidified environment at room temperature. The participants were instructed to rinse and clean their appliances using only a toothbrush. They were informed not to brush the area containing the enamel block.

\section{Aciduric experiment}

After completion of each treatment period, both the OBs and NOBs were used for the aciduric experiments. The blocks were re-covered with nail varnish (remineralization area, RA), leaving half the window uncovered (aciduric experiment area, AEA). For the aciduric experiment, the blocks were immersed in $50 \mathrm{ml}$ demineralizing solution ( $0.1 \mathrm{M}$ lactic acid, $0.3 \mathrm{mM}$ hydroxyapatite, $1 \%$ carboxymethyl cellulose sodium salt, $\mathrm{pH} 4.7$ ) at $37^{\circ} \mathrm{C}$ for 3 days.

\section{Contact microradiography}

Both sets of blocks were dehydrated in a graded series of ethanol and embedded in polyester resin (Rigolac; Nisshin EM Co. Ltd, Tokyo, Japan). Ground cross sections $100 \mu \mathrm{m}$ in thickness were prepared from the enamel blocks. Contact microradiograms (CMRs) of the ground sections were obtained using a soft X-ray generator (Softex CMR-3; Softex Co., Ltd, Tokyo, Japan) with a $20-\mu \mathrm{m}$ Ni filter operated at an accelerating voltage of $15 \mathrm{kV}$ and a specimen current of $3 \mathrm{~mA}$ for $10 \mathrm{~min}$ with $\mathrm{Cu}-\mathrm{K} \alpha$. An aluminum 
Yasuo Miakeet al.: Remineralization and Acid Resistance of Enamel by Calcified Seaweed
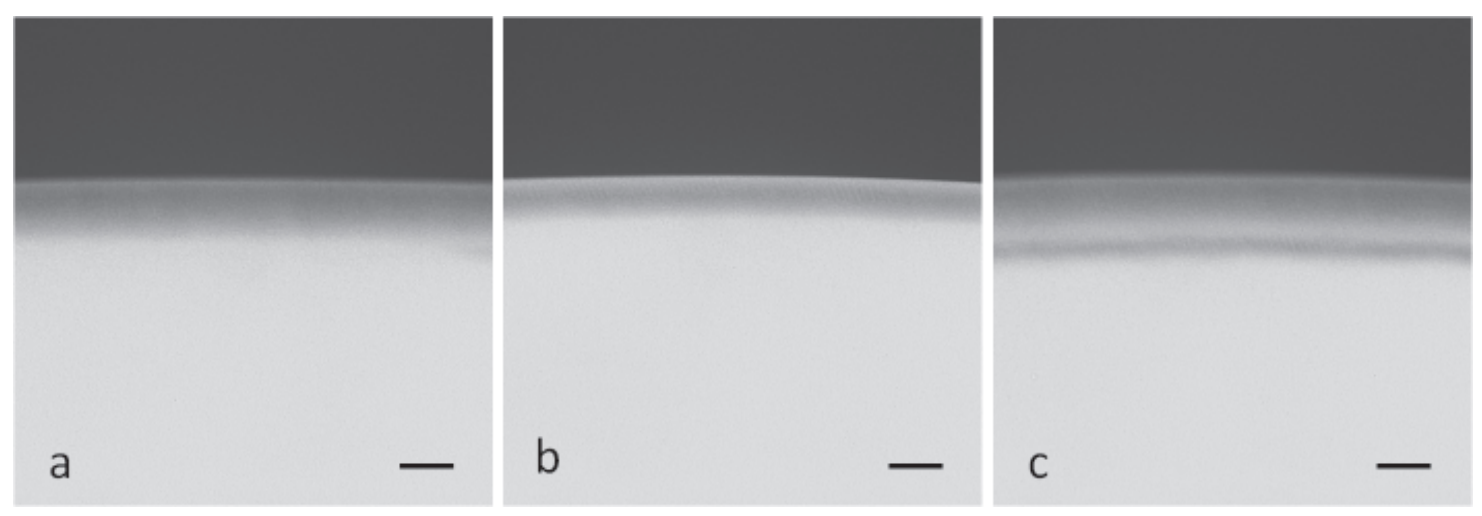

Figure1. Example from CS group. Demineralized area (a), remineralized area (b) and aciduric experiment area (c)Bars:100 $\mu \mathrm{m}$
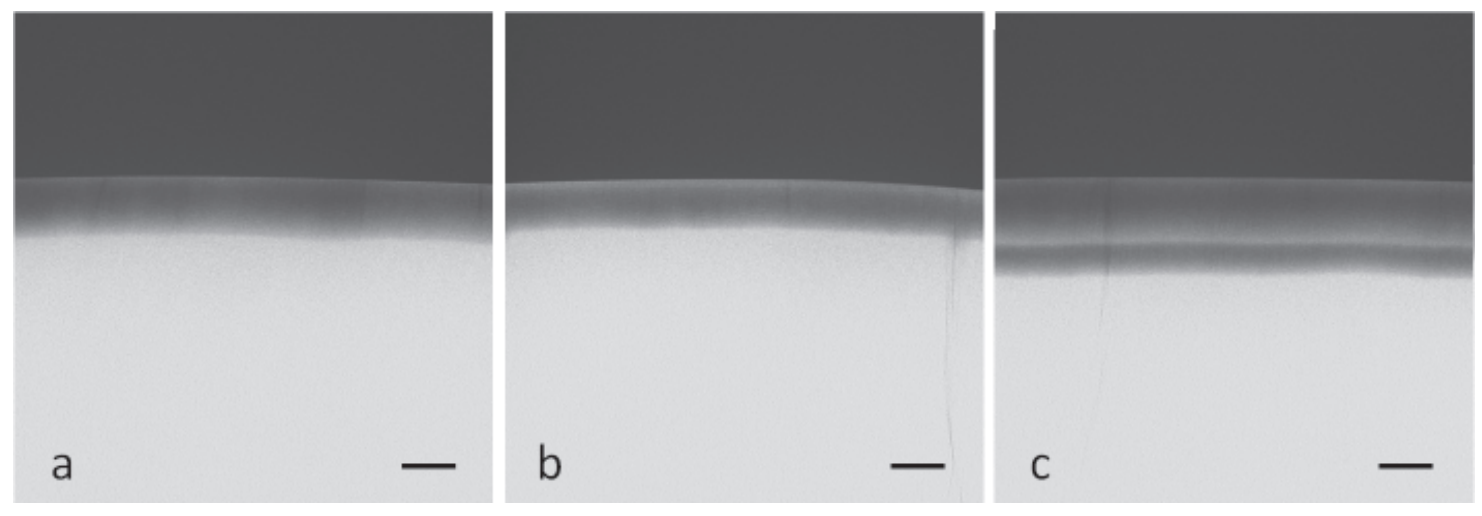

Figure 2. Example from non-CS group. Demineralized area (a), remineralized area (b) and aciduric experiment area (c). Bars:100 $\mu \mathrm{m}$

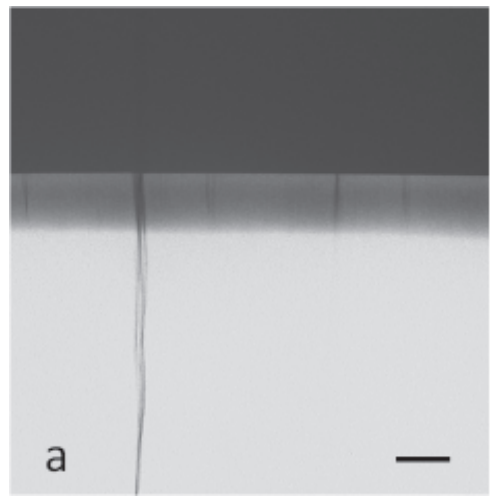

Figure 3. Example from DE group. Demineralized area (a)

Aciduric experiment area.(c) Bars: $100 \mu \mathrm{m}$

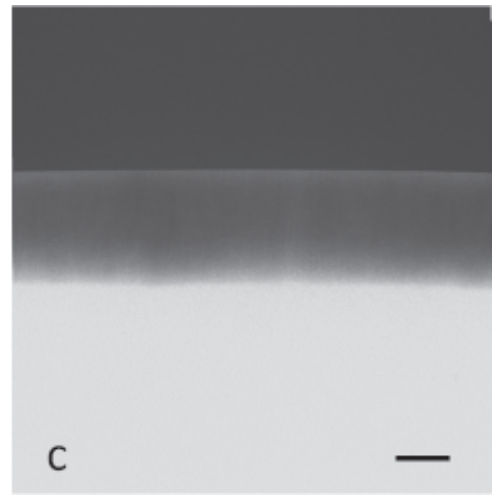

step wedge (20 $\mu \mathrm{m} \times 20$ steps) was placed on an X-ray glass film plate (HRP-SN-2; Konica Minolta, Tokyo, Japan) with the sample for image analysis. The glass film plate was developed (D-19; Kodak, USA) for $5 \mathrm{~min}$ at $20^{\circ} \mathrm{C}$, rinsed with tap water and then fixed (Fujifix; Fuji Film, Tokyo, Japan) for $5 \mathrm{~min}$ at $20^{\circ} \mathrm{C}$.

\section{Image analysis}

The CMR images were obtained using the Image Analyze System (HC-2500/OL; Olympus, Tokyo, Japan: HDS-N1; Hiroya, Tokyo, Japan). Only areas free of artifacts and cracks were selected for the analysis. Each digital image was made in 256 level grey scale with a width of $50 \mu \mathrm{m}$ and depth of $300 \mu \mathrm{m}$ (from resin base to sound enamel). Three cross sections of each area (DA, RA, and AEA) were measured and the mean obtained. The demineralization rate (mineral loss value, $\Delta \mathrm{Z}$ ) of each experimental area was calculated in terms of aluminum equivalence by setting the photographic density of the film (not involving the sample) to $0 \%$ and that of sound enamel to $100 \%$. Linear regression was used to convert the grey value data into values of equivalent thickness of aluminum. The thickness of each section was measured and the percent mineral data of the demineralization rate computed using the equation of Angmar et al. ${ }^{23)}$. Lesion depth (Ld) was defined as the distance from the surface to the point in the lesion at which the mineral content was larger than $95 \%$ of 
Table 1 - Results of Ld and $\Delta \mathrm{Z}$. Table shows Ld (lesion depth) and $\Delta \mathrm{Z}$ (mineral loss value). Demineralization area (DA), Remineralization area (RA) and aciduric experiment area (AEA). Data represent mean \pm S.D. $(n=10)$.

\begin{tabular}{lcccccc}
\hline & \multicolumn{3}{c}{$\operatorname{Ld}(\mu \mathrm{m})$} & \multicolumn{4}{c}{$\Delta \mathrm{Z}(\mathrm{vol} \% \cdot \mu \mathrm{m})$} \\
\hline & DA & RA & AEA & DA & RA & AEA \\
CS & $106.9 \pm 8.1$ & $100.4 \pm 21.2$ & $120.0 \pm 13.2$ & $3751 \pm 474$ & $3072 \pm 700$ & $4583 \pm 500$ \\
non-CS & $106.8 \pm 18.9$ & $103.0 \pm 18.1$ & $129.1 \pm 11.7$ & $3988 \pm 1014$ & $3539 \pm 669$ & $5584 \pm 514$ \\
DE & $106.7 \pm 19.2$ & - & $131.3 \pm 12.3$ & $3595 \pm 510$ & - & $6142 \pm 813$ \\
\hline
\end{tabular}

Table 2 - Results of \%R and \%A. Table shows \%R (remineralization rate) and \%A (aciduric rate). The \%A value shows demineralizing progress, resulting in large negative value.

\begin{tabular}{lcc}
\hline & $\% \mathrm{R}$ & $\% \mathrm{~A}$ \\
\hline CS & 18.09 & -22.18 \\
non-CS & 11.26 & -40.03 \\
DE & - & -70.82 \\
\hline
\end{tabular}

the mineral content in sound enamel. The percent mineral profile of each enamel block's demineralized and remineralized lesions was compared with that of the sound enamel between lesions of the same specimen. In addition, owing to exfoliation of or damage to the nail varnish in some cases, measurement was carried out in a total of 10 specimens in each group. The $\Delta \mathrm{Zs}$ of the $\mathrm{DA}(\Delta \mathrm{Zd})$, $\mathrm{RA}(\Delta \mathrm{Zr})$ and AEA ( $\Delta \mathrm{Za}$ ) were measured. The $\Delta \mathrm{Z}$ and Ld data were analyzed using an analysis of variance (ANOVA) with the multiple comparison Tukey test. The critical level for alpha was set at 0.05 . A remineralization rate $(\% \mathrm{R})$ and aciduric rate $(\% \mathrm{~A})$ were calculated according to the following formula ${ }^{24)}$.

$$
\begin{aligned}
& \% \mathrm{R}=(1-\Delta \mathrm{Zr} / \Delta \mathrm{Zd}) \times 100 \\
& \% \mathrm{~A}=(1-\Delta \mathrm{Za} / \Delta \mathrm{Zd}) \times 100
\end{aligned}
$$

\section{Electron probe microanalyzer examination}

After coating the ground sections with a thin layer of carbon, qualitative and aspect analyses of $\mathrm{Ca}, \mathrm{P}, \mathrm{Mg}$ and $\mathrm{F}$ ions were carried out using an electron probe microanalyzer (EPMA) (JXA-8200; JEOL, Tokyo, Japan). The observations described above were carried out on the sound, DA, RA and AEA.

\section{CMR images}

\section{Results}

In the OBs, DA, RA and AEA were observed. In the NOBs, DA and AEA were observed. Figures 1, 2 and 3 show examples from the CS group (Fig. 1), non-CS group (Fig. 2) and demineralization experiments (DE) group (Fig. 3) respectively.

In the DA, although the enamel showed demineralization to an almost uniform depth, the degree of mineralization at the surface was moderately high (Figs. 1a, 2a, 3a). In the RA, a high degree of remineralization was observed at both the surface and at a deep layer, and the width of the demineralized area decreased in comparison with that of the DA. The middle layer was also uniformly remineralized (Figs. 1b, 2b). In the CS and non-CS in aciduric experiment, further demineralization was observed beneath the original demineralized layer, and overall depth of demineralization increased. A thin, highly mineralized layer was observed between the two demineralized layers (Figs. 1c, 2c). In the DE in the aciduric experiment, the width of the demineralized layer increased, but no thin, mineralized layer was observed between the other two layers (Fig. 3c).

\section{Image analysis}

Lesion depth $(\mu \mathrm{m})$ and $\Delta \mathrm{Z}(\mathrm{vol} \%, \mu \mathrm{m})$ on each CMR image were determined (Table 1).

No significant difference in $\mathrm{Ld}$ or $\Delta \mathrm{Z}$ was obtained between each DA and RA. A significant difference was observed in Ld in the aciduric experiment between the CS group and DE $(\mathrm{p}<0.05)$. A significant difference was observed in $\Delta \mathrm{Z}$ in the aciduric experiment between the CS group and the non-CS group and DE $(\mathrm{p}<0.05)$.

Table 2 shows calculation of remineralization rate $(\% \mathrm{R})$ and aciduric rate (\%A).

Degree of remineralization in CS group was higher than that in non-CS group. Furthermore, aciduric rate in CS group was higher than that in non-CS group. Results showed that acid resistance was strengthened in CS group.

\section{EPMA analysis}

Qualitative analysis of the surface region in all samples revealed the presence of $\mathrm{Ca}$ and $\mathrm{P}$, but not that of $\mathrm{Mg}$ or F. While the aspect analysis revealed that the distribution density of $\mathrm{Ca}$ and $\mathrm{P}$ decreased in DA and RA compared with that in sound enamel, no $\mathrm{Mg}$ or $\mathrm{F}$ were detected. No conspicuous difference in distribution density was observed between each aciduric experiment.

\section{Discussion}

In this study, in the aciduric experiment, a thin, highly 
Yasuo Miakeet al.: Remineralization and Acid Resistance of Enamel by Calcified Seaweed

mineralized layer was observed between the demineralized layers by CMR. This layer showed that highly aciduric crystals had formed. This indicates that xylitol played a major role in the remineralization observed here. It is known that xylitol enhances enamel remineralization ${ }^{15}$. Moreover, in an earlier study, the use of xylitol with fluoride was shown to increase remineralization and decrease demineralization ${ }^{25}$. Calcified seaweed contains large amounts of $\mathrm{Ca}, \mathrm{P}$ and $\mathrm{F}$, all of which would enter the saliva through the process of chewing. It is know that even small quantities of $F$, in particular, affect crystal nucleation and growth. These ions act synergistically, causing an increase in crystallinity or crystal growth. In the aciduric experiment in this study, chewing calcified seaweed-containing gum significantly increased acid resistance. This indicates that the $\mathrm{Ca}, \mathrm{P}$ and $\mathrm{F}$ contained in the gum exerted a strong effect on remineralization.

On the other hand, calcified seaweed also contains large amounts of Mg. It has been reported that $\mathrm{Mg}$ increased the solubility of apatite crystals in enamel ${ }^{26,27}$. It is possible that $\mathrm{Mg}$ affected crystallinity in the calcified seaweed-containing gum experiment here. However, aciduric enhancement occurred in this experiment, suggesting that the action of $\mathrm{Ca}, \mathrm{P}$ and $\mathrm{F}$ was greater than that of the Mg.

In the calcified seaweed-containing gum experiment, $\mathrm{Ca}$ and $\mathrm{P}$ were detected by qualitative and aspect analyses; $\mathrm{Mg}$ and $\mathrm{F}$, however, were not detected by EPMA. It is known that even very small amounts of $\mathrm{F}$ exert a considerable effect on apatite crystal growth. Therefore, it seems that $\mathrm{F}$ was effective, even at levels below those that could be detected by EPMA.

In conclusion, the results of this study suggest that chewing calcified seaweed-containing gum significantly increases remineralization and acid resistance due to the action of xylitol and $\mathrm{F}$. This indicates that calcified seaweed-containing gum would be effective in the restoration of initial carious lesions and caries prevention.

\section{Acknowledgements}

This study was supported by a grant from the Central Laboratory, Lotte Co., Ltd. and in part by the Oral Science Center, Tokyo Dental College. We appreciate the participation of the volunteer subjects. The authors would like to thank Associate Professor Jeremy Williams, Tokyo Dental College, for his assistance with the English of the manuscript.

\section{Reference}

1. Yanagisawa T, Miake Y, Saeki Y and Takahashi M. Remineralization in enamel caries and restoration of carious lesions by enhanced remineralization induced by saliva and xylitol. Dentistry in Japan 39: 208-215, 2003

2. Dreizen S, Mann AW, Cline JK and Tom DS. The buffer capacity of saliva as a measure of dental caries. J Dent Res
25: 213-222, 1946

3. Carey CM, Gregory TM, Tatevossian A and Vogel GL; The buffer capacity of single-site, resting, human dental-plaque fluid. Arch Oral Biol 33: 487-492, 1988

4. Hamon CB and Klebanoff SJ. A peroxidase-mediated, streptococcus mitis-dependent antimicrobial system in saliva. J Exp Med 137: 438-450, 1973

5. Bowen WH. Defense mechanisms in the mouth and their possible role in the prevention of dental caries. J Oral Pathol 3: 266-278, 1974

6. Shannon IL, Suddick RP and Dowd FJ Jr. Saliva: Composition and secretion. Monogra Oral Sci 2: 1-103, 1974

7. Shaw L, Murray JJ, Burchell CK and Best JS. Calcium and phosphorus content of plaque and saliva in relation to dental caries. Caries Res 17: 543-548, 1983

8. Dawes $\mathrm{C}$ and Kubieniec K. The effects of prolonged gum chewing on salivary flow rate and composition. Arch Oral Biol 49: 665-669, 2004

9. Dawes $\mathrm{C}$ and Macpherson LM. Effects of nine different chewinggums and lozenges on salivary flow rate and $\mathrm{pH}$. Caries Res26: 176-182, 1992

10. Edgar WM and Geddes DA. Chewing gum and dental healtha review. Br Dent J 24: 173-177, 1990

11. Makinen KK. Sweeteners and promotion of dental caries. Special reference to xylitol. Oral Health78: 57-66, 1988

12. Makinen KK, Makinen PL, Pape HR Jr, Allen P, Bennett CA, Isokangas PJ and Isotupa KP. Stabilisation of rampant caries: polyol gums and arrest of dentine caries in two longterm cohort studies in young subjects. Int Dent J 45: 93107, 1995

13. Makinen KK and Soderling E. Solubility of calcium salts, enamel, and hydroxyapatite in aqueous solution of simple carbohydrates. Calcif Tissue Int 36: 64-71, 1984

14. Arends J, Smits M, Ruben JL and Christoffersen J. Combined effect of xylitol and fluoride on enamel demineralization in vitro. Caries Res 24: 256-257, 1990

15. Miake Y, Saeki Y, Takahashi M and Yanagisawa T. Remineralization effect of xylitol on demineralized enamel. J Electron Microsc52: 471-476, 2003

16. Zimmer S, Jahn KR and Barthel CR. Recommendations for the use of fluoride in caries prevention. Oral Health Prev Dent1: 45-51, 2003

17. Thaweboon S, Nakornchai S, Miake Y, Yanagisawa T, Thaweboon B, Soo-ampon S and Lexomboon D. Remineralization of enamel subsurface lesions by xylitol chewing gum containing funoran and calcium hydrogenphosphate. Southeast Asian J Trop Med Public Health40: 345-353, 2009

18. Meyer-Lueckel $\mathrm{H}$ and Tschoppe P. Effect of fluoride gels and mouthrinses in combination with saliva substitutes on 
J.Hard Tissue Biology Vol. 21(3):315-320, 2012

demineralised bovine enamel in vitro. J Dent 38: 641-647, 2010

19. Cochrane NJ, Cai F, Huq NL, Burrow MF and Reynolds EC. New approaches to enhanced remineralization of tooth enamel. J Dent Res 89: 1187-1197, 2010

20. Yamazaki T, Miake Y, Ishikawa T, Hiruma N and Yanagisawa T. The effect of calcified seaweed on remineralization of artificially demineralized dentin. J Hard Tissue Biol 20: 1116, 2011

21. Miake Y, Nozue S, Moriguchi M, Yamazaki T, Sawada T and Yanagisawa T. The ability of chewing gum with calcified seaweed in preventing demineralization of tooth surfaces. $\mathrm{J}$ Hard Tissue Biol 20: 87-92, 2011

22. White DJ. Use of synthetic polymer geld for artificial carious lesion preparation. Caries Res 21: 228-242, 1987
23. Angmar B, Carlstrome D and Glas JE. Studies on the ultrastructure of dental enamel. IV. The mineralization of normal human enamel. J Ultrastruct Res 8: 12-23, 1963

24. Reynolds EC. Remineralization of enamel subsurface lesions by casein phosphopeptide-stabilized calcium phosphate solutions. J Dent Res 76: 1587-1595, 1997

25. Amaechi BT, Higham SM and Edgar WM. Caries inhibiting and remineralizing effect of xylitol in vitro. J Oral Sci 41: 71-76, 1999

26. Boulet M, Marier JR and Rose D. Effect of magnesium on formation of calcium phosphate precipitates. Arch Biochem Biophys 96: 629-636, 1962

27. Okazaki M, Takahashi J and Kimura H. Unstable behavior of magnesium-containing hydroxyapatites. Caries Res 20: 324-331, 1986 